Article

\title{
Plate-Type Acoustic Metamaterials: Experimental Evaluation of a Modular Large-Scale Design for Low-Frequency Noise Control
}

\author{
Linus Yinn Leng Ang ${ }^{1,2, * \mathbb{C}}$, Yong Khiang Koh ${ }^{2}$ and Heow Pueh Lee ${ }^{1} \mathbb{C}$ \\ 1 Department of Mechanical Engineering, National University of Singapore, 9 Engineering Drive 1, \\ Singapore 117575, Singapore; mpeleehp@nus.edu.sg \\ 2 Kinetics Design and Manufacturing, ST Engineering Land Systems Ltd., 249 Jalan Boon Lay, \\ Singapore 619523, Singapore; kohyk@stengg.com \\ * Correspondence: ylang@u.nus.edu or anglinus@stengg.com
}

Received: 8 February 2019; Accepted: 22 March 2019; Published: 1 April 2019

\begin{abstract}
For industrial applications, the scalability of a finalised design is an important factor to consider. The scaling process of typical membrane-type acoustic metamaterials may pose manufacturing challenges such as stress uniformity of the membrane and spatial consistency of the platelet. These challenges could be addressed by plate-type acoustic metamaterials with an internal tonraum resonator. By adopting the concept of modularity in a large-scale design (or meta-panel), the acoustical performance of different specimen configurations could be scaled and modularly combined. This study justifies the viability of two meta-panel configurations for low-frequency (80-500 Hz) noise control. The meta-panels were shown to be superior to two commercially available noise barriers at 80-500 Hz. This superiority was substantiated when the sound transmission class (STC) and the outdoor-indoor transmission class (OITC) were compared. The meta-panels were also shown to provide an average noise reduction of $22.7-27.4 \mathrm{~dB}$ at $80-400 \mathrm{~Hz}$ when evaluated in different noise environments-traffic noise, aircraft flyby noise, and construction noise. Consequently, the meta-panel may be further developed and optimised to obtain a design that is lightweight and yet has good acoustical performance at below $500 \mathrm{~Hz}$, which is the frequency content of most problematic noises.
\end{abstract}

Keywords: large-scale; plate-type acoustic metamaterial; resonator; modular; noise control; sound transmission loss; noise reduction; environmental noise

\section{Introduction}

Membrane-type acoustic metamaterials have been studied extensively over the past decade [1-12] because they could exhibit good acoustical performance at below $1 \mathrm{kHz}$ while being compact and lightweight. To date, the literature provides many different designs-ranging from simple to complex constructions - that may be considered as a benchmark for further development. This class of acoustic metamaterial is usually realised by adhering the edges of a pretensioned membrane to a rigid frame. To pretension the membrane, a mechanism could be designed to stretch the membrane edges uniformly over a prescribed distance [1]. Alternatively, epoxy could be applied along the membrane edges in which stretching is achieved by thermal curing [4]. Evidently, to produce the specimen in large quantities, the assembly process would likely require a huge amount of time and effort. This issue may be addressed by considering either a honeycomb panel [13] or a perforated panel [14] as the frame. The acoustical performance is characterised solely by the resonant behaviour of the membrane in which the frequency bandwidths of good acoustical performance are achieved at around the anti-resonance frequencies, while the frequency bandwidths of poor acoustical performance are obtained at around 
the resonance frequencies. By varying the dimensions, the material properties, and the pretension of the membrane, the frequency bandwidths of good acoustical performance could be adjusted to coincide with the frequency range of the unwanted noise. Consequently, noise control is achieved. The membrane could also be attached with a highly dense platelet to introduce additional tuning parameters. The list of tuning parameters may be extended. For example, an additional support structure could be implemented to suppress specific eigenmodes of the membrane [8], or a perforated membrane could be considered to introduce the effect of a Helmholtz resonator [2].

Knowing that membrane-type acoustic metamaterials could potentially be considered in the industry for noise control, it is imperative that the finalised small-scale designs are extendable. Of course, it would be ideal if the large-scale design could exhibit the same acoustical performance achieved during the small-scale study. Considering how typical designs are realised, manufacturing challenges may be faced during the scaling process. These challenges, for example, include stress relaxation and stress uniformity of the membrane, and spatial consistency of the platelet on the membrane. Moreover, if numerous platelets were considered, the scaling process would be laborious. Huang et al. [15] reported that the challenges could be faced even for small-scale designs. Hence, avenues should be explored to address the challenges and improve the scalability of membrane-type acoustic metamaterials.

Recently, numerous works [16-20] have shown that a plastic film can be considered in place of the elastic membrane to eliminate the challenges pertaining to stress relaxation and stress uniformity. Since there is negligible pretension in the film, the membrane-type acoustic metamaterial is transformed into another class of acoustic metamaterial known as the plate-type acoustic metamaterial. Despite this transformation, the acoustical performance is still characterised solely by the resonant behaviour of the film. Again, if a platelet was considered to modify the resonant behaviour, the challenge relating to spatial consistency would remain unresolved. To address this challenge, Ang et al. [21,22] proposed that the design could adopt a double-leaf configuration with an internal tonraum resonator (i.e., two cavities connected via an orifice) so that the objective of having more tuning parameters could still be met without attaching platelets.

Over the past five years, researchers $[1,23,24]$ have shown growing interest in large-scale membrane-type acoustic metamaterials despite the aforementioned challenges. However, large-scale plate-type acoustic metamaterials have yet to be studied extensively, evident from the literature in which limited works $[20,25,26]$ can be found. Varanasi et al. [20,25] proposed a large-scale design $\left(1.22 \times 1.22 \mathrm{~m}^{2}\right)$ that had close resemblance to a plate-type acoustic metamaterial. The design was fabricated from a continuous acrylic panel in which the unit cells were realised by varying the thickness at different regions of the panel. Although numerous configurations were considered, none had dissimilar unit cells on the same panel to study whether they could collectively contribute to the acoustical performance of the panel. Ang et al. [26] filled in the research gap with a large-scale design $\left(994 \times 994 \mathrm{~mm}^{2}\right)$ that adopted the concept of modularity for customisable acoustical performance. The proof-of-concept design-also referred to as the meta-panel—was realised by slotting specimens with two different configurations (without and with resonator) into the periodic cutouts on a host structure. By varying the distribution percentage of the specimens on the host structure, the acoustical performance of the meta-panel was shown to be collectively contributed by the acoustical performance of each specimen configuration. Additionally, the acoustical performance of the meta-panel was found to be insensitive to how the specimens were oriented or arranged on the host structure. The findings suggest that the meta-panel could potentially be considered in the industry for noise control.

This study aims to justify whether the meta-panel could indeed be viable for noise control in the industry by investigating two selected meta-panel configurations reported previously [26] in greater detail. This investigation compares the acoustical performance with that of two commercially available noise barriers and evaluates the acoustical performance in three different noise environments-traffic noise, aircraft flyby noise, and construction noise. Section 2 presents the design details of the two meta-panel configurations and the experimental methods. Section 3 presents the results. Section 4 
discusses how the findings could provide avenues for future research. Section 5 concludes the study based on the observations.

\section{Materials and Methods}

This section first presents the two selected meta-panel configurations (Section 2.1) before moving on to discuss the experimental methods (Section 2.2). To minimise repetition, only the differences between this study and the previous work [26] are discussed. Nonetheless, crucial information is still provided.

\subsection{Meta-Panel Configurations}

The two meta-panel configurations were selected because they exhibited the widest frequency bandwidth of good acoustical performance at low frequencies $(<500 \mathrm{~Hz})$ compared to that of the other configurations [26]. Both configurations were solely occupied by specimens with resonator (Figure 1a), which were previously investigated in a small-scale study [21,22]. The difference between the two configurations is the material of the host structure in which one was made of aluminium and the other was made of plywood.

The host structure consisted of an inner panel and two outer skins (Figure 1b). The former was designed with $10 \times 10$ cutouts ( $22 \mathrm{~mm}$ apart) that were used to house the specimens, while the latter served as a flange to constrain the specimens from falling out of the cutouts. To achieve the flange, the $10 \times 10$ cutouts on the outer skins were $32 \mathrm{~mm}$ apart. The inner panel had a thickness $(20 \mathrm{~mm})$ that matched that of the specimens, while the outer skins were specified with a thickness $(3 \mathrm{~mm})$ so that they would not be too flimsy especially for the case of plywood. Fasteners were used to assemble the inner panel and the outer skins at 32 points. This number of points is inclusive of the eight points used by the handles, which were replaced by countersunk screws during the measurements.

Each specimen was assembled from two Mylar films (127 $\mu \mathrm{m}$ thick) and three printed components. The printed components-two outer frames and one inner frame (Figure 1a)—were produced from polylactide filament via fused deposition modelling (MOMENT Moment S). To assemble the specimen, a layer of adhesive tape (Nitto Denko No. 500) was first applied on each side of the inner frame. The width of the tape $(70 \mathrm{~mm})$ was specially customised to be larger than the lengths of the inner frame $(60 \mathrm{~mm})$ to avoid discontinuity, which could result in air leakages from the cavities. Each side of the inner frame was then adhered with a film that was simply laid flat on a tabletop. This approach minimised the presence of pretension - an essential attribute of plate-type acoustic metamaterials. The excess tapes and films were then removed by cutting. Finally, one outer frame (applied with a layer of adhesive tape on one side) was adhered to each film to emulate a pinned boundary condition along the film edges. The orifice was specified at the centre of the partition for ease of numerical modelling and realisation. The orifice diameter $(5 \mathrm{~mm})$ was specified such that the influence of the viscous boundary layer would be minimal as shown in the previous work [22].

\subsection{Experimental Methods}

This section first presents how the sound transmission class (STC) and the outdoor-indoor transmission class (OITC) of the meta-panels were determined for comparison with that of two commercially available noise barriers (Sections 2.2.1 and 2.2.2, respectively). Next, this section discusses how the meta-panels were evaluated to determine the noise reduction in three different noise environments (Sections 2.2.3). 
(a)

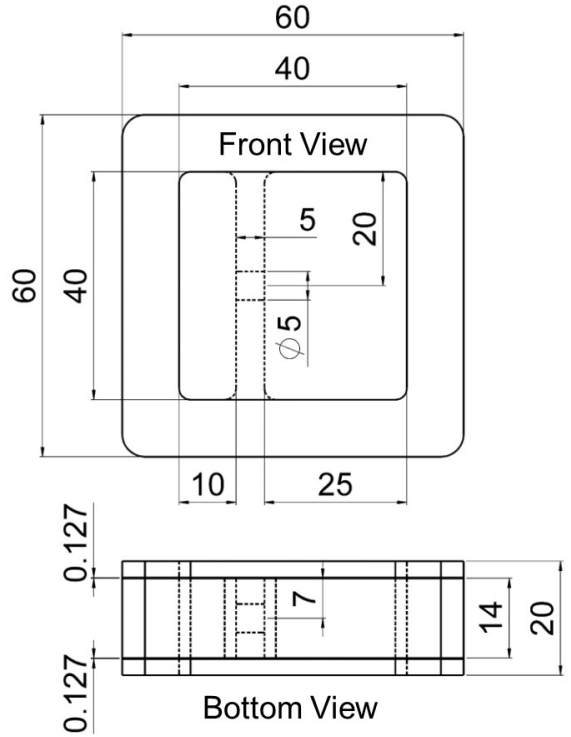

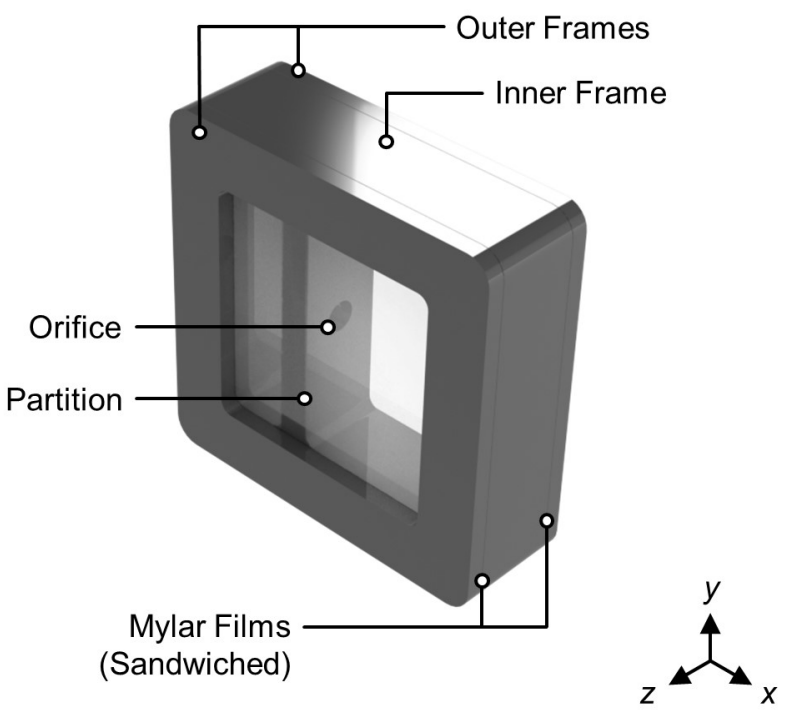

(Sandwiched)

(b)

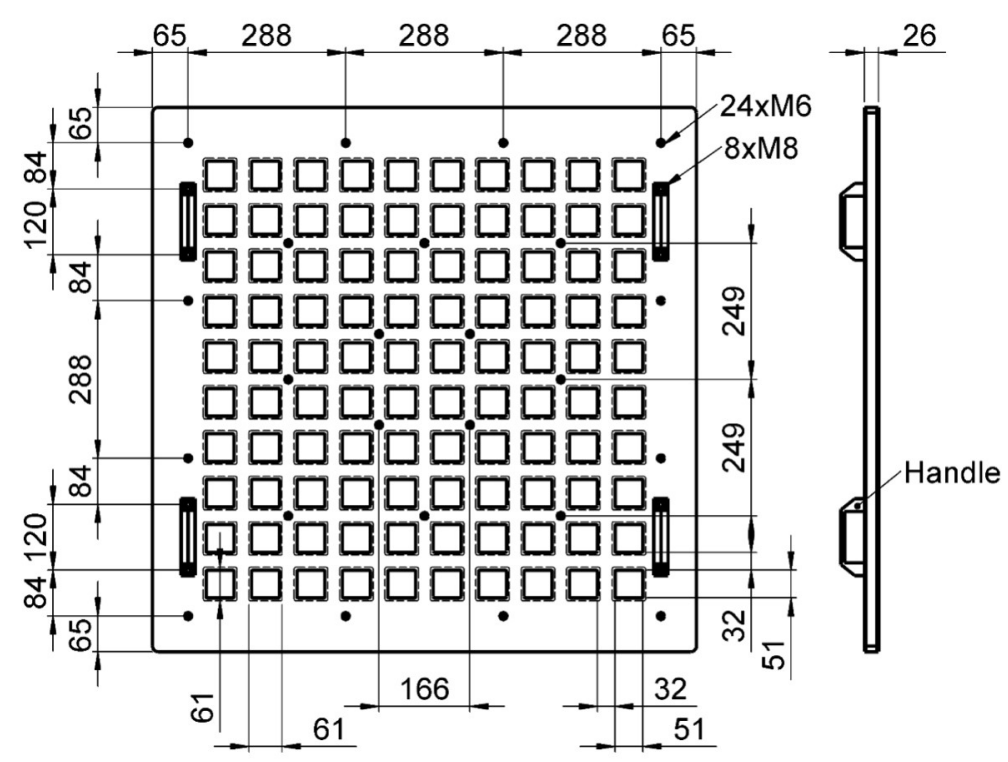

Figure 1. (a) Technical drawing (front and bottom views) and three-dimensional rendering of the specimen with resonator. (b) Technical drawing (front and side views) of the host structure. Hidden lines indicate the cutouts on the inner panel. All dimensions are in millimetres.

\subsubsection{Sound Transmission Class}

The sound transmission loss (STL) of the meta-panels was first determined to obtain the STC through post-processing. The measurements were conducted according to ASTM E90 [27] in two reverberation chambers. The source and the receiver chambers had a volume of 65.5 and $81.1 \mathrm{~m}^{3}$, respectively, and a surface area of 97.2 and $109.5 \mathrm{~m}^{2}$, respectively. The chambers were separated by a commercial acoustic wall, which was designed with an opening to mount the meta-panel from the receiver chamber. The opening had a surface area of $1 \mathrm{~m}^{2}$ and a depth of $10 \mathrm{~cm}$. Three toggle clamps were used to secure each edge of the meta-panel. As the meta-panel was $2.6 \mathrm{~cm}$ thick (Figure 1b), a wooden frame with a cross-sectional area of $8 \times 2.5 \mathrm{~cm}^{2}$ was required to transfer the clamping forces onto the edges of the meta-panel. A flange was designed around the opening to prevent further translation of the meta-panel towards the source chamber. Gasket strips were adhered along the flange to minimise sound leakages. Figure 2 shows the described set-up when viewed from each chamber. 

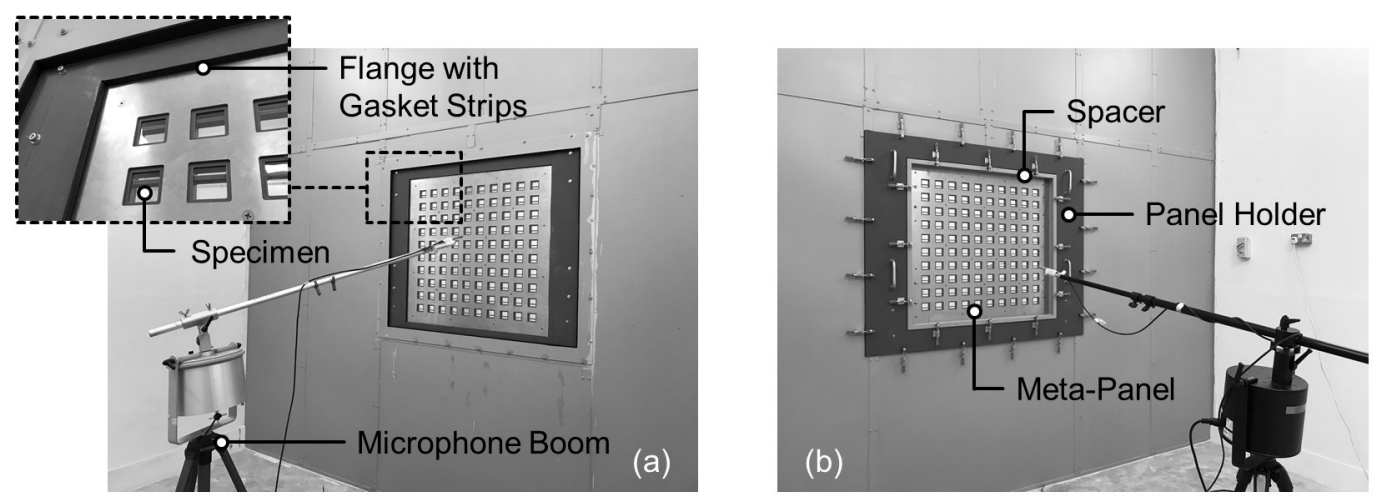

Figure 2. View of the test set-up in the (a) source and the (b) receiver chambers.

Pink noise was produced by a noise generator (Brüel \& Kjær Type 2734) and transmitted into the source chamber by a pair of loudspeakers (Yamaha DXR15). Each loudspeaker was positioned at the trihedral corner furthest away from the panel holder. The noise signal was ensured to be loud enough such that the sound pressure level (SPL) at each one-third octave band frequency ( $80 \mathrm{~Hz}$ to $5 \mathrm{kHz}$ ) was more than $10 \mathrm{~dB}$ above the background noise in the receiver chamber. Consequently, background noise correction was not required. The SPL in each chamber was measured by a microphone boom (Norsonic Nor265). As recommended in ASTM E90 [27], at least six measurements (30 s each) were collected for averaging. Subsequently, the measurements were post-processed to calculate the STL by [27]

$$
S T L=\bar{L}_{S}-\bar{L}_{r}+10 \log _{10} \frac{S}{A_{r}}
$$

where $\bar{L}_{S}$ and $\bar{L}_{r}$ denote the average SPL in the source and the receiver chambers, respectively; $S$ denotes the surface area of the meta-panel; and $A_{r}$ denotes the measured absorption area of the receiver chamber, which is given by [28]

$$
A_{r}=55.26 \frac{V_{r}}{\bar{T}_{60} c_{0}}
$$

where $V_{r}$ denotes the volume of the receiver chamber $\left(81.1 \mathrm{~m}^{3}\right) ; \bar{T}_{60}$ denotes the average reverberation time; and $c_{0}$ denotes the speed of sound in air at $24.7^{\circ} \mathrm{C}$, which was the average temperature of the receiver chamber. In Equations (1) and (2), all variables are frequency-dependent except for $S, V_{r}$, and $c_{0}$.

From Equation (1), the STC was then determined based on the requirements and the reference curve given in ASTM E413 [29]. The requirements include (1) the deficiency at each one-third octave band frequency from $125 \mathrm{~Hz}$ to $4 \mathrm{kHz}$ must not exceed $8 \mathrm{~dB}$ and (2) the sum of all deficiencies must be less than or equal to $32 \mathrm{~dB}$. A deficiency is a point in which the measured STL is less than that of the reference curve. If both requirements are met, the STC will be equal to the STL of the reference curve at $500 \mathrm{~Hz}$.

To include experimental uncertainty, the results are presented alongside the $95 \%$ confidence intervals $\Delta X$ calculated by [27]

$$
\Delta X=C s_{X}
$$

where $C$ denotes the constant corresponding to $N$ measurements $\left(C=1.05\right.$ for $N=6$ [27]) and $s_{X}$ denotes the standard deviation of the variable $X$. For instance, $X$ can be substituted with $A_{r}$ to obtain the $95 \%$ confidence intervals for Equation (2). Only $s_{X}$ is frequency-dependent. For Equation (1), the $95 \%$ confidence intervals $\triangle S T L$ were calculated by [27]

$$
\Delta S T L=\left[\Delta L_{s}^{2}+\Delta L_{r}^{2}+18.9\left(\Delta A_{r} / A_{r}\right)^{2}\right]^{0.5}
$$


where $\Delta L_{s}$ and $\Delta L_{r}$ denote the $95 \%$ confidence intervals of the SPL in the source and the receiver chambers, respectively.

\subsubsection{Outdoor-Indoor Transmission Class}

Since 1955, the STC has been used to classify building elements that could be treated as some sort of noise barrier with a single-value rating based on the overall acoustical performance from $125 \mathrm{~Hz}$ to $4 \mathrm{kHz}$ [27]. Typical building elements include exterior/interior walls, doors, and windows, not to mention soundproofing canvases. Developed in 1990, the OITC classifies the same group of building elements in a similar way as the STC but places more emphasis on the low-frequency range $(80 \mathrm{~Hz}$ to $4 \mathrm{kHz}$ ) [30]. The classification considers down to $80 \mathrm{~Hz}$ instead of $125 \mathrm{~Hz}$, which is the case for the STC. The upper frequency limit remains the same at $4 \mathrm{kHz}$. According to ASTM E1332 [30], the OITC was designed to evaluate how effective the partition could mitigate outdoor ground and air transportation noises that are predominantly low-frequency $(<500 \mathrm{~Hz})$.

To obtain a reliable OITC, the noise source should exhibit a frequency spectrum that follows the trend of the reference curve given in ASTM E1332 [30]. The reference curve was derived by averaging the frequency spectra of aircraft takeoff noise, train passing noise, and traffic noise. In this study, the pink noise was considered as the noise source because its frequency spectrum could follow closely to the trend of the reference curve (Figure 3). As opposed to the reference curve used to determine the STC, adjustment of the curve to fit the measured STL curve based on deficiencies is not required. The OITC is mathematically expressed by [30]

$$
\text { OITC }=100.13-10 \log _{10} \sum_{f=80}^{4000} 10^{\left(L_{r e f, f}+L_{A, f}-S T L_{f}\right) / 10}
$$

where $L_{r e f}$ denotes the SPL of the reference curve; $L_{A}$ denotes the A-weighting correction factors; $S T L$ denotes the values obtained from Equation (1); and the subscript $f$ denotes that the variable is dependent on the one-third octave band frequency from $80 \mathrm{~Hz}$ to $4 \mathrm{kHz}$.

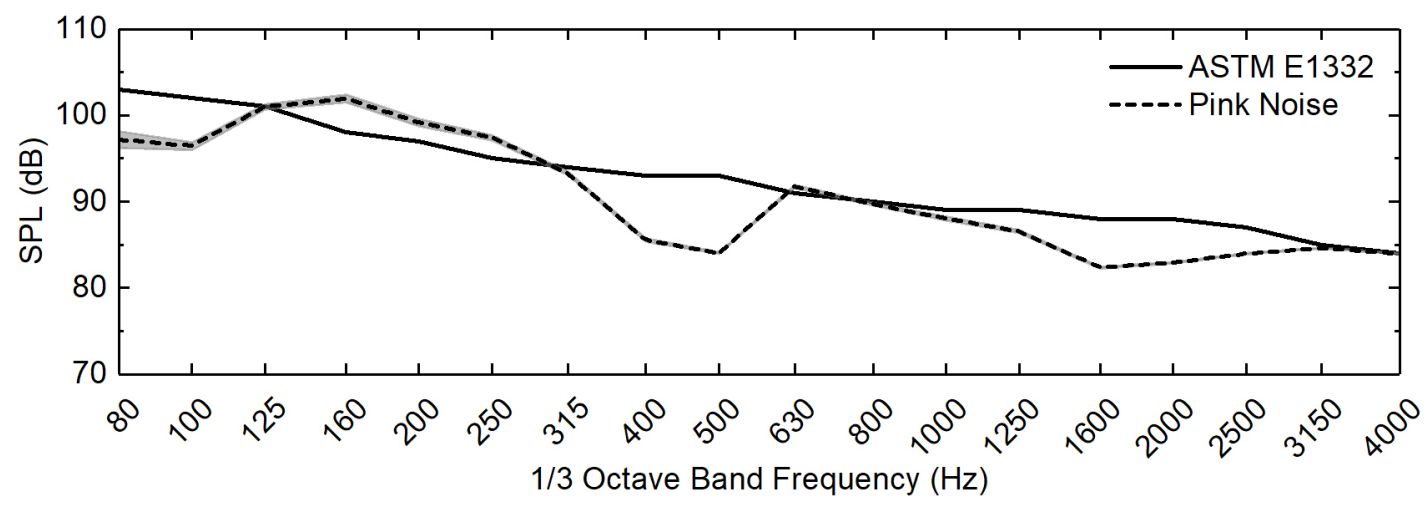

Figure 3. Comparison between the reference curve given in ASTM E1332 [30] and the measured SPL curve of the pink noise in the source chamber. Shaded region denotes the limits of the experimental uncertainty at a confidence level of $95 \%$.

\subsubsection{Noise Reduction}

As mentioned at the end of Section 1, three different noise environments were considered-traffic noise, aircraft flyby noise, and construction noise. The first two noises were on-site recordings in Singapore, while the latter was downloaded from the Internet. These noises were considered because they are commonly experienced in Singapore. The test set-up was identical to that shown in Figure 2. Imagine the receiver chamber as an office room or a residential unit and the source chamber as the exterior environment polluted by each considered noise. The meta-panel can be imagined as the 
building element-a see-through panel, for example-separating the two domains. Based on this set-up, it is valid to determine how well the meta-panel would perform in terms of noise reduction $L_{n r}$ as given by

$$
L_{n r}=\bar{L}_{s}-\bar{L}_{r}
$$

As defined in Equation (1), $\bar{L}_{S}$ and $\bar{L}_{r}$ denote the average SPL in the source and the receiver chambers, respectively, and are frequency-dependent.

\section{Results}

This section first presents the results based on the comparison between the meta-panels and the two commercially available noise barriers (Section 3.1). Next, the acoustical performance of the meta-panels in three different noise environments is discussed (Section 3.2). The detailed analysis of the specimen's characteristic was performed and can be found in the previous works $[21,22,26]$.

\subsection{Comparison with Commercially Available Noise Barriers}

The scope of this comparative study is limited to the consideration of commercially available noise barriers found in Singapore-ANB 80 Steel and PEB Super Galum Steel (Figure 4). To allow a fair comparison, it is crucial to be aware of the thickness and the surface density of the meta-panels and the noise barriers (Table 1). This section first compares the measured STL curves (Section 3.1.1) before moving on to compare the STC and the OITC (Sections 3.1.2 and 3.1.3, respectively).
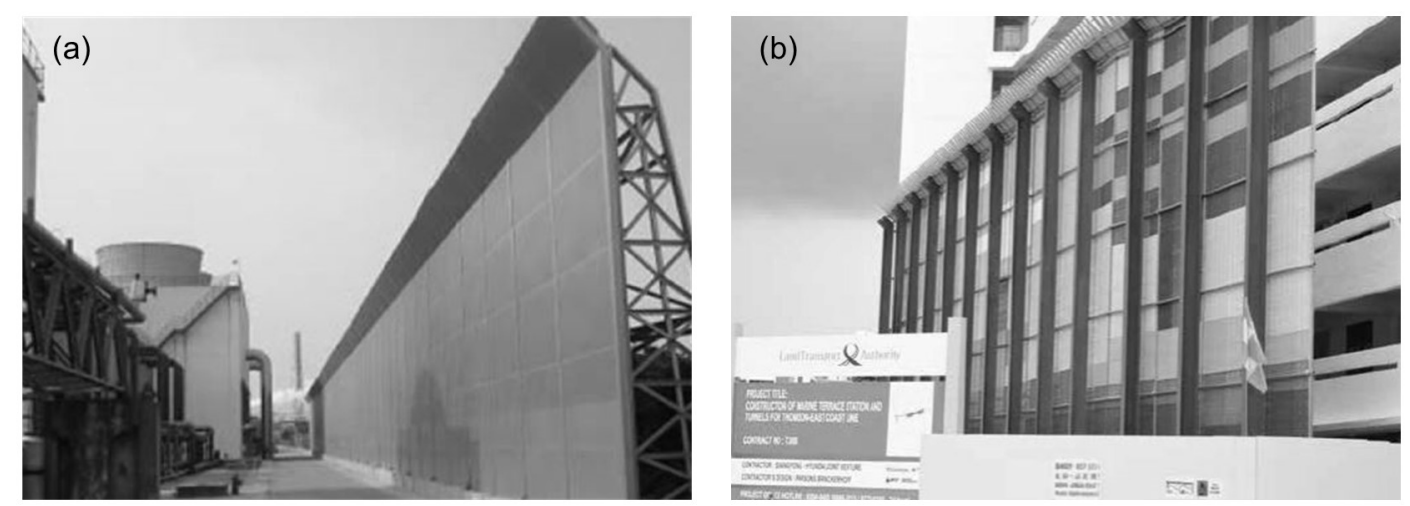

Figure 4. Commercially available noise barriers found in Singapore-(a) ANB 80 Steel and (b) PEB Super Galum Steel. Retrieved from the respective manufacturer's brochures.

Table 1. Thickness and surface density of the meta-panels-aluminium and wooden-and the noise barriers-ANB 80 Steel and PEB Super Galum Steel.

\begin{tabular}{lcc}
\hline Product Name & Thickness $(\mathbf{m m})$ & Surface Density $\left(\mathbf{k g} / \mathbf{m}^{2}\right)$ \\
\hline Aluminium Meta-Panel & 26 & 51.1 \\
Wooden Meta-Panel $_{\text {ANB 80 Steel }}^{*}$ & 26 & 16.4 \\
PEB Super Galum Steel $^{*}$ & 80 & 20.0 \\
\hline
\end{tabular}

* From manufacturer's brochure.

\subsubsection{Sound Transmission Loss}

To facilitate the analysis, the difference in STL was calculated by subtracting the STL curves of the noise barriers from that of each meta-panel (Figure 5). A positive difference indicates superiority of each meta-panel; a negative difference indicates inferiority of each meta-panel. The measured STL curves of the noise barriers are provided in Figure A1 in Appendix A to supplement this discussion. 
At 80-500 Hz, both meta-panels were superior to the noise barriers. The aluminium meta-panel performed better than the ANB 80 Steel and the PEB Super Galum Steel by up to $18.4 \mathrm{~dB}$ at $200 \mathrm{~Hz}$ and up to $25.4 \mathrm{~dB}$ at $315 \mathrm{~Hz}$, respectively (Figure 5a). For the wooden meta-panel, it performed better than the ANB 80 Steel and the PEB Super Galum Steel by up to $10.4 \mathrm{~dB}$ at $400 \mathrm{~Hz}$ and up to $19.1 \mathrm{~dB}$ at $125 \mathrm{~Hz}$, respectively (Figure 5b). At this frequency range, the superiority of the meta-panels to the noise barriers was attributed to the stiffness region of the films, preventing the fundamental frequency of the entire structure from adversely affecting the acoustical performance. This phenomenon was justified in the previous work [26]. For the noise barriers, there was no additional feature to minimise the impact of the structural resonance, providing a possible explanation to the poorer acoustical performance.
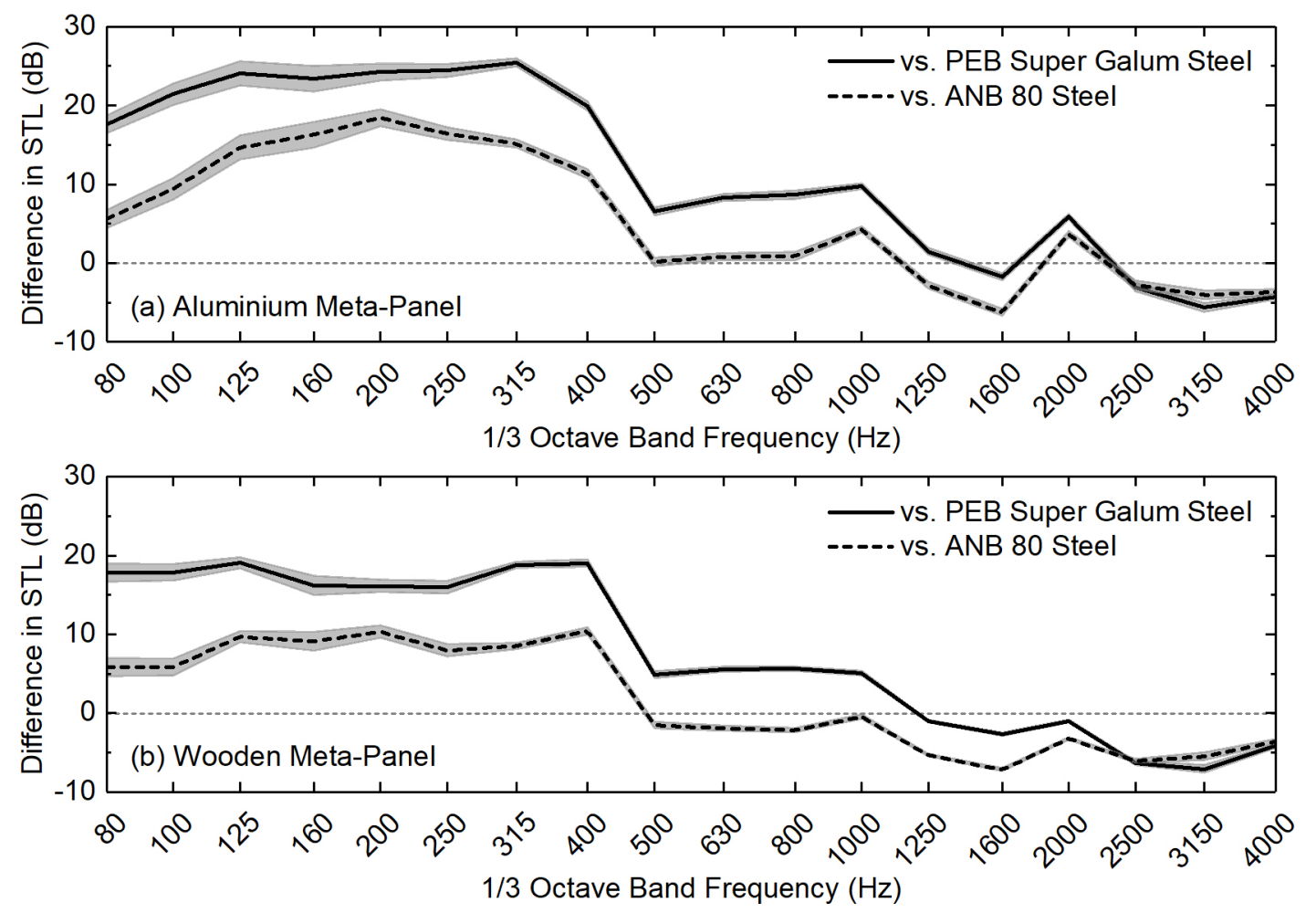

Figure 5. Difference in STL between the meta-panels-(a) aluminium and (b) wooden-and the noise barriers-PEB Super Galum Steel and ANB 80 Steel. Shaded region denotes the limits of the experimental uncertainty at a confidence level of $95 \%$.

The superiority of both meta-panels extended further from 500 to $1250 \mathrm{~Hz}$ when compared against the PEB Super Galum Steel. The aluminium and the wooden meta-panels performed better by up to $9.7 \mathrm{~dB}$ at $1 \mathrm{kHz}$ and up to $5.1 \mathrm{~dB}$ at $1 \mathrm{kHz}$, respectively. The maximum difference in STL at $1 \mathrm{kHz}$ was contributed by one of the "noise shield" effects of the films. The "noise shield" effect refers to a phenomenon in which the displacement profile of the second film was nearly motionless relative to the first film. As such, the second film functioned like a "noise shield", minimising sound transmission. Again, this phenomenon was justified in the previous work [26]. Conversely, at the same frequency range (500-1250 Hz), the acoustical performance of each meta-panel was comparable to that of the ANB 80 Steel. The aluminium meta-panel performed better by only $0.1-0.9 \mathrm{~dB}$ except at $1 \mathrm{kHz}(4.2 \mathrm{~dB})$, while the wooden meta-panel fared poorer by $0.4-2.2 \mathrm{~dB}$.

Unfortunately, the superiority of the meta-panels did not extend further into the high-frequency range $(1.25-4 \mathrm{kHz})$, indicating that the meta-panels would not be ideal for high-frequency noise control. The wooden meta-panel performed poorer than the ANB 80 Steel and the PEB Super Galum Steel by up to $7.2 \mathrm{~dB}$ at $1.6 \mathrm{kHz}$ and up to $7.1 \mathrm{~dB}$ at $3.15 \mathrm{kHz}$, respectively (Figure $5 \mathrm{~b}$ ). For the aluminium meta-panel, it performed poorer than the ANB 80 Steel and the PEB Super Galum Steel by up to 
$6.3 \mathrm{~dB}$ at $1.6 \mathrm{kHz}$ and up to $5.7 \mathrm{~dB}$ at $3.15 \mathrm{kHz}$, respectively (Figure 5a). Despite the poorer acoustical performance at high frequencies, an improvement in STL was observed at $2 \mathrm{kHz}$ due to the "noise shield" effect of the films. In particular, this effect provided the aluminium meta-panel with a positive difference in STL over the noise barriers at the same frequency. In this case, the aluminium meta-panel performed better than the ANB 80 Steel and the PEB Super Galum Steel by up to 3.7 and $5.9 \mathrm{~dB}$, respectively. Despite the acoustical drawback at high frequencies, it may not pose any concern because most problematic noises fall at around and/or below $1 \mathrm{kHz}$.

From the above analysis, it is justifiable that the meta-panels may provide acoustical advantage over the noise barriers if the primary aim is to mitigate low-frequency noise $(<500 \mathrm{~Hz})$. Depending on the configuration, the meta-panels could also mitigate mid-frequency noise $(0.5-1 \mathrm{kHz})$. Of course, it can be argued that the significant difference in STL between the aluminium meta-panel and the noise barriers could also be contributed by its high surface density (Table 1), which was 2.46 and 4.87 times higher than that of the ANB 80 Steel and the PEB Super Galum Steel, respectively. To refute this argument, it may be more convincing to compare the products that were of similar surface density. Based on the surface densities given in Table 1, the wooden meta-panel and the ANB 80 Steel-16.4 and $20.0 \mathrm{~kg} / \mathrm{m}^{2}$, respectively-may provide a fairer comparison. In fact, the surface density of the latter was higher than the former by $7.0 \%$, giving it acoustical advantage over the wooden meta-panel based on the concept of the mass law [31]. This advantage is indeed the case in which the ANB 80 Steel performed better than the wooden meta-panel at $0.5-4 \mathrm{kHz}$ (Figure 5b). According to the mass law [31], the higher the surface density, the higher the STL at a given frequency. However, at $80-500 \mathrm{~Hz}$, the same noise barrier (ANB 80 Steel) performed poorer than the wooden meta-panel despite its higher surface density. At this frequency range, the wooden meta-panel benefited from the dynamic response of the specimens slotted within it. This comparison implies that the meta-panel, regardless of material, could still be superior to the noise barriers even with comparable surface densities.

\subsubsection{Sound Transmission Class}

At this juncture, the comparison of the measured STL curves showed that the meta-panels could perform better than the noise barriers especially if the primary focus is to mitigate low-frequency noise $(<500 \mathrm{~Hz})$. In the industry, the STC is often preferred to rate how effective the product is in noise mitigation. Therefore, to be more comprehensive, this section extends the previous section to compare the STC of the meta-panels and the noise barriers (Table 2).

Table 2. STC of the meta-panels-aluminium and wooden-and the noise barriers-ANB 80 Steel and PEB Super Galum Steel.

\begin{tabular}{lc}
\hline Product Name & STC \\
\hline Aluminium Meta-Panel & 36 \\
Wooden Meta-Panel & 33 \\
ANB 80 Steel & 33 \\
PEB Super Galum Steel & 25 \\
\hline
\end{tabular}

Expectedly, the aluminium meta-panel was superior to the noise barriers with a STC of 36-3 and 11 points higher than that of the ANB 80 Steel (STC of 33) and the PEB Super Galum Steel (STC of 25), respectively. For the wooden meta-panel, it fared slightly poorer than the aluminium meta-panel by 3 points with a STC of 33. This rating was 8 points higher than that of the PEB Super Galum Steel. Recall the comparison in the previous section between the wooden meta-panel and the ANB 80 Steel. Even though the latter performed better than the former across a wider frequency range $(0.5-4 \mathrm{kHz}$ as opposed to $80-500 \mathrm{~Hz}$ ), the STC showed that both products were in fact comparable in acoustical performance. The reason is because the STC emphasises more on the low-frequency range, which is the frequency content of most problematic noises [29]. Knowing that both products were identical in STC, it may be preferable to consider the wooden meta-panel over the ANB 80 Steel because of its higher 
STL at 80-500 Hz, not to mention its lower surface density (Table 1). Moreover, the present design of the meta-panels is only a proof-of-concept and has yet to undergo design optimisation. Consequently, the decision-making process should be easier.

\subsubsection{Outdoor-Indoor Transmission Class}

To justify that the meta-panels had acoustical advantage over the noise barriers especially at low frequencies (80-500 Hz), this section compares their OITC with that of the noise barriers (Table 3).

Table 3. OITC of the meta-panels-aluminium and wooden-and the noise barriers-ANB 80 Steel and PEB Super Galum Steel.

\begin{tabular}{lc}
\hline Product Name & OITC \\
\hline Aluminium Meta-Panel & 34 \\
Wooden Meta-Panel & 31 \\
ANB 80 Steel & 26 \\
PEB Super Galum Steel & 18 \\
\hline
\end{tabular}

When evaluated based on the OITC instead of the STC, the ANB 80 Steel and the PEB Super Galum Steel performed even poorer by 7 points. The former dropped from a STC of 33 to an OITC of 26; the latter dropped from a STC of 25 to an OITC of 18. This drop in rating suggests that both noise barriers were inherently poorer in acoustical performance at low frequencies (80-500 Hz) - a shortcoming that was amplified when the OITC was considered. As such, the aluminium meta-panel became even more superior to the noise barriers with an OITC of 34-12 and 16 points higher than that of the ANB 80 Steel and the PEB Super Galum Steel, respectively. The significant drop in rating difference from 3 (STC) to 12 (OITC) showed by the ANB 80 Steel suggests that it would not be ideal in low-frequency noise environments $(<500 \mathrm{~Hz})$. For the wooden meta-panel, it performed poorer than the aluminium meta-panel by 3 points - the same number of points when comparing between the STC.

In Section 3.1.2, the comparison between the wooden meta-panel and the ANB 80 Steel showed that they were comparable in acoustical performance based on the STC even though the latter performed better than the former across a wider frequency range (0.5-4 kHz in contrast to $80-500 \mathrm{~Hz}$ ). However, when evaluated based on the OITC instead of the STC, the acoustical advantage of the wooden meta-panel at low frequencies $(80-500 \mathrm{~Hz})$ was clearly demonstrated in which it performed better than the ANB 80 Steel by 5 points with an OITC of 31. This comparison substantiates the recommendation in the last paragraph of Section 3.1.2 stating that it may be preferable to consider the wooden meta-panel over the ANB 80 Steel because of its higher STL at 80-500 Hz and its lower surface density (Table 1).

\subsection{Acoustical Performance in Different Noise Environments}

Having shown the acoustical advantage of the meta-panels at low frequencies (80-500 Hz), it was of interest to evaluate their acoustical performance in three different noise environments-traffic noise, aircraft flyby noise, and construction noise-as shown in Figure 6. The measured SPL curves of the noise environments are provided in Figure A2 in Appendix B to supplement this discussion.

Both meta-panels exhibited consistent acoustical performance in the three different noise environments. The noise reduction curves followed closely to the overall trend of the STL curves that were reported previously for the same configuration (i.e., solely occupied by specimens with resonator) [26]. Focusing on the low-frequency range (80-400 Hz), the aluminium meta-panel provided a noise reduction of 18.4-30.9 dB depending on the noise environment (Figure 6a). For the wooden meta-panel, it achieved a noise reduction of 18.3-26.9 dB depending on the noise environment (Figure 6b). Averaging over the same frequency range, the aluminium and the wooden meta-panels provided a noise reduction of 27.4 and $22.7 \mathrm{~dB}$, respectively. This observation suggests that the meta-panel, regardless of material, could significantly reduce the noise exposure of the occupants. 

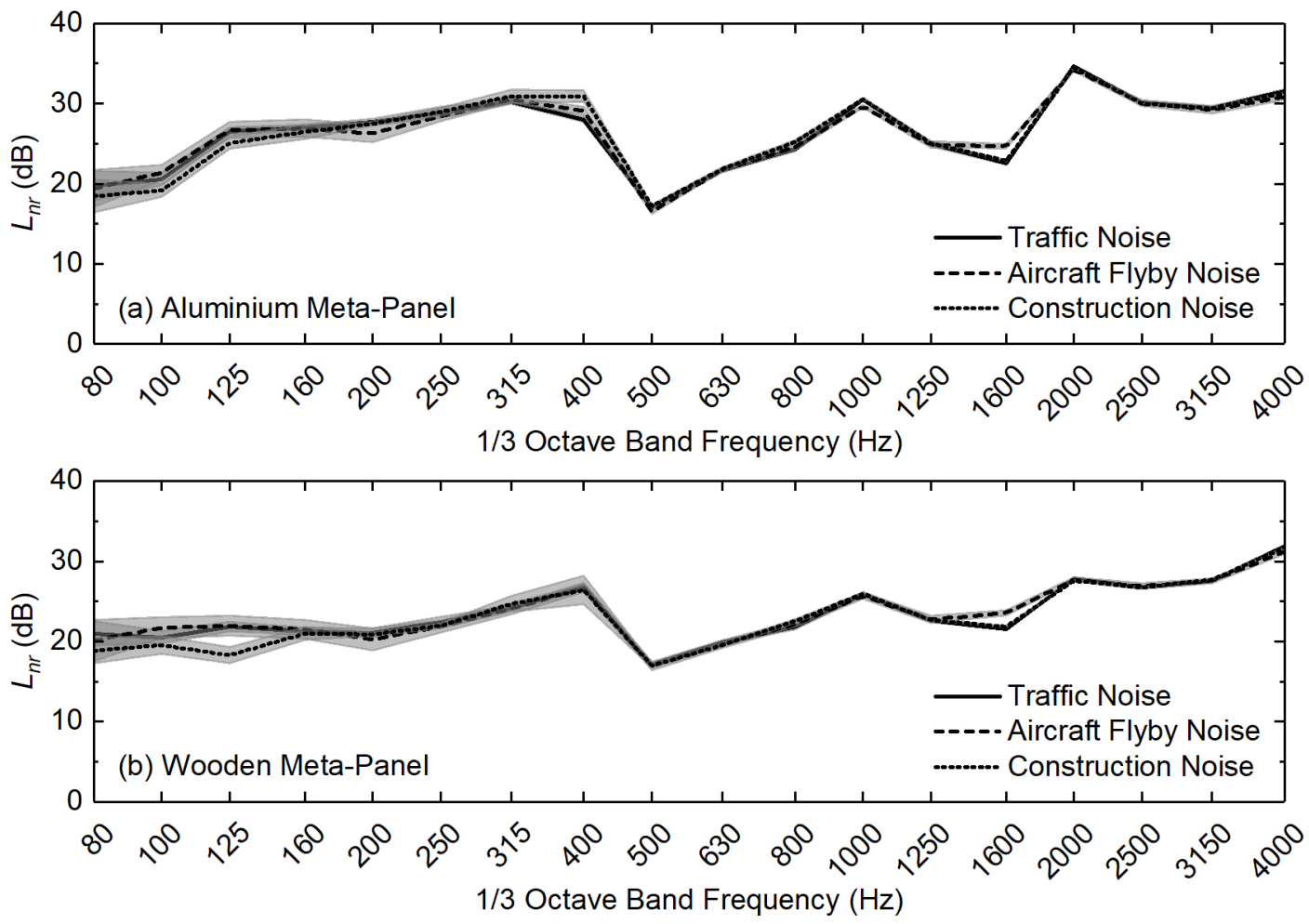

Figure 6. Noise reduction $L_{n r}$ curves of the meta-panels-(a) aluminium and (b) wooden-due to traffic noise, aircraft flyby noise, and construction noise. Shaded region denotes the limits of the experimental uncertainty at a confidence level of $95 \%$.

\section{Discussion}

Naturally, being a proof-of-concept, it is no doubt that further development and optimisation are necessary to arrive at a meta-panel design suitable for the noise environment of interest. This section discusses some of the avenues for future research.

The influence of different weather conditions on the meta-panel has yet to be considered. In Singapore, the weather conditions include heavy rain, strong wind, and hot sun. If the meta-panel is considered for outdoor applications-as a noise barrier, for example-the specimens and the host structure must be designed with adequate structural integrity. A protective feature may be required to prevent the films from excessive deflection, which could alter the resonant behaviour of the films and influence the characteristic of the meta-panel. For example, the protective feature could simply be a continuous panel fastened to each side of the meta-panel to enclose the specimens. Consequently, the overall acoustical performance of the meta-panel may also be improved based on the understanding of the mass law. The protective feature could also serve to prevent surface damage of the films and dust accumulation on the films. In actual environments, the former could be caused by foreign objects, while the latter is inevitable. If both factors are not considered, the durability of the meta-panel will be affected, not to mention its reliability in noise mitigation.

The concept of modularity could provide the meta-panel with versatility and aesthetics. By having a library of uniquely designed specimens, the meta-panel could be configured such that its acoustical performance would be optimised for the noise environment of interest. The specimens could also be colour-coded to categorise them according to their acoustical performance. Since the acoustical performance of the meta-panel was previously shown [26] to be insensitive to the distribution and the arrangement of the specimens, both factors could be varied to form a pixel art, providing aesthetics to the meta-panel. 
Being a design that is highly customisable for different noise environments is no doubt advantageous especially in terms of implementation cost. As the host structure does not need to be replaced during the change in configuration, the cost incurred from the customisation should only be dependent on the cost of producing new specimens. In this study, the production cost of each specimen was approximately $S \$ 4$. This amount may be reduced further if the specimens are produced in large quantities via conventional manufacturing techniques.

Lastly, as the scope of this study was limited to experimental evaluation, it is essential to develop a numerical model of the meta-panel so that the desired acoustical performance could be predicted prior to fabrication. Consequently, high computational power will be expected.

\section{Conclusions}

To reiterate, the purpose of this study was to justify whether the aluminium and the wooden meta-panels solely occupied by specimens with resonator could indeed be viable for noise control in the industry. By comparing the STL with that of two commercially available noise barriers, the meta-panels were shown to be superior at low frequencies $(80-500 \mathrm{~Hz})$. This superiority was substantiated when the STC and the OITC were compared. The meta-panels were then evaluated in three different noise environments, showing that they could provide an average noise reduction of 27.4 and $22.7 \mathrm{~dB}$, respectively, at $80-400 \mathrm{~Hz}$. In conclusion, the meta-panels were justified to be viable for low-frequency $(80-500 \mathrm{~Hz})$ noise control in the industry. Being a proof-of-concept design, there is a large room for improvement to obtain a meta-panel that is lightweight and yet has good acoustical performance at low-frequency range $(<500 \mathrm{~Hz})$, which is the frequency content of most problematic noises.

Author Contributions: L.Y.L.A. conceived the idea, designed the methodology, fabricated the specimens, performed the measurements/simulations, analysed the data, and wrote/revised the manuscript. Y.K.K. and H.P.L. provided feedback and edited/approved the manuscript for submission.

Funding: This research was funded by the Singapore Economic Development Board (EDB) and ST Engineering Land Systems Ltd. under the EDB Industrial Postgraduate Program (EDB-IPP) (Grant No. COY-15-IPP/120005).

Acknowledgments: The authors acknowledge the advice from Samuel Lee and the support from Kok Seng Cheng during the design and the experimental phases of this research, respectively.

Conflicts of Interest: The authors declare no conflict of interest.

\section{Abbreviations}

The following abbreviations are used in this manuscript:

STC Sound Transmission Class

OITC Outdoor-Indoor Transmission Class

STL Sound Transmission Loss

SPL Sound Pressure Level

\section{Appendix A. Measured STL Curves of the Noise Barriers}

Figure A1 compares the measured STL curves of the aluminium and the wooden meta-panels to that of the noise barriers. The measured STL curves of both noise barriers were extracted from the respective manufacturer's brochures. As the STL at $80 \mathrm{~Hz}$ was not available for both noise barriers, it was taken as the same STL at $100 \mathrm{~Hz}$. The STL curves were used to calculate the difference in STL between the meta-panels and the noise barriers (Figure 5). 


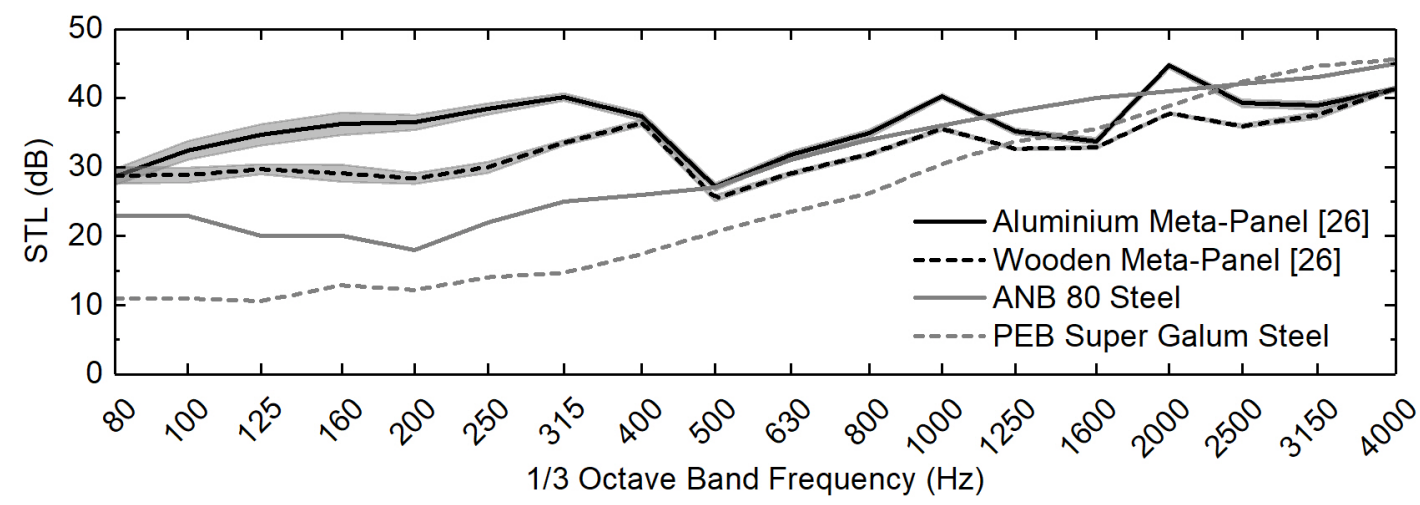

Figure A1. Measured STL curves of the aluminium and the wooden meta-panels compared to that of the noise barriers-ANB 80 Steel and PEB Super Galum Steel. Shaded region denotes the limits of the experimental uncertainty at a confidence level of $95 \%$.

\section{Appendix B. Measured SPL Curves of the Noise Environments}

Figure A2 shows the measured SPL curves of the noise environments in the source chamber. The values of the SPL curves were used to calculate the noise reduction $L_{n r}$ curves (Figure 6).

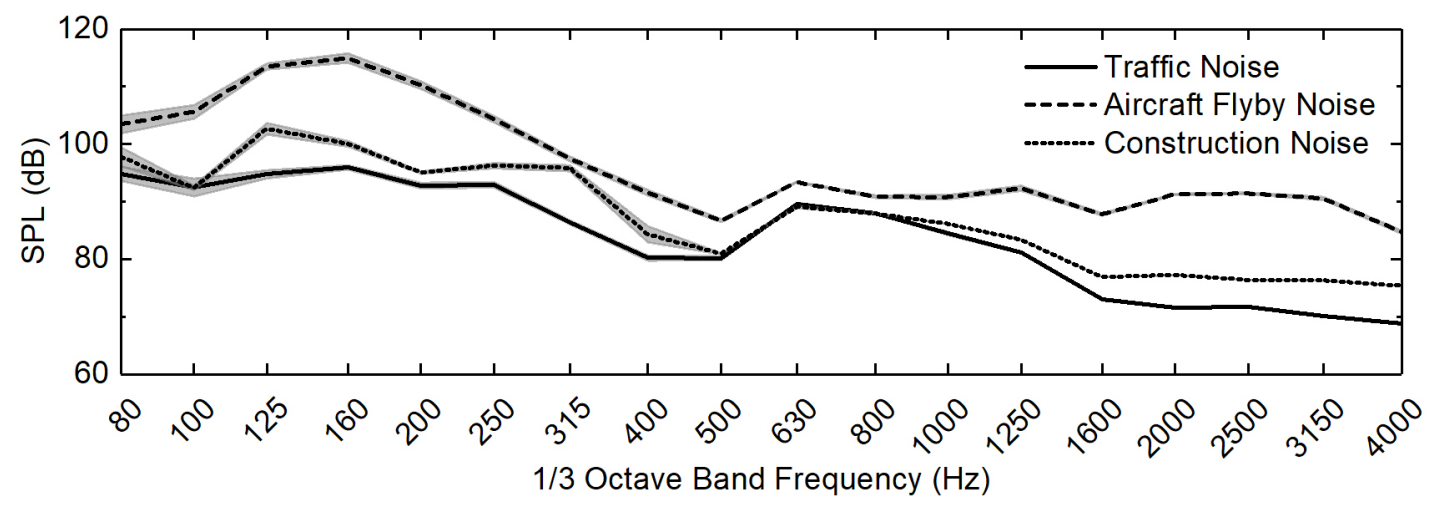

Figure A2. Measured SPL curves of the three different noise environments-traffic noise, aircraft flyby noise, and construction noise-in the source chamber. Shaded region denotes the limits of the experimental uncertainty at a confidence level of $95 \%$.

\section{References}

1. Ang, L.Y.L.; Koh, Y.K.; Lee, H.P. Broadband sound transmission loss of a large-scale membrane-type acoustic metamaterial for low-frequency noise control. Appl. Phys. Lett. 2017, 111, 041903. [CrossRef]

2. Langfeldt, F.; Kemsies, H.; Gleine, W.; von Estorff, O. Perforated membrane-type acoustic metamaterials. Phys. Lett. A 2017, 381, 1457-1462. [CrossRef]

3. Mei, J.; Ma, G.; Yang, M.; Yang, Z.; Wen, W.; Sheng, P. Dark acoustic metamaterials as super absorbers for low-frequency sound. Nat. Commun. 2012, 3, 756. [CrossRef] [PubMed]

4. Naify, C.J.; Chang, C.M.; McKnight, G.; Nutt, S.R. Transmission loss and dynamic response of membrane-type locally resonant acoustic metamaterials. J. Appl. Phys. 2010, 108, 114905. [CrossRef]

5. Naify, C.J.; Chang, C.M.; McKnight, G.; Scheulen, F.; Nutt, S.R. Membrane-type metamaterials: Transmission loss of multi-celled arrays. J. Appl. Phys. 2011, 109, 104902. [CrossRef]

6. Naify, C.J.; Chang, C.M.; McKnight, G.; Nutt, S.R. Transmission loss of membrane-type acoustic metamaterials with coaxial ring masses. J. Appl. Phys. 2011, 110, 124903. [CrossRef]

7. Naify, C.J.; Chang, C.M.; McKnight, G.; Nutt, S.R. Scaling of membrane-type locally resonant acoustic metamaterial arrays. J. Acoust. Soc. Am. 2012, 132, 2784-2792. [CrossRef] 
8. Wang, X.; Zhao, H.; Luo, X.; Huang, Z. Membrane-constrained acoustic metamaterials for low-frequency sound insulation. Appl. Phys. Lett. 2016, 108, 041905. [CrossRef]

9. Wang, X.; Luo, X.; Zhao, H.; Huang, Z. Acoustic perfect absorption and broadband insulation achieved by double-zero metamaterials. Appl. Phys. Lett. 2018, 112, 021901. [CrossRef]

10. Yang, Z.; Mei, J.; Yang, M.; Chan, N.H.; Sheng, P. Membrane-type acoustic metamaterial with negative dynamic mass. Phys. Rev. Lett. 2008, 101, 204301. [CrossRef]

11. Yang, Z.; Dai, H.M.; Chan, N.H.; Ma, G.; Sheng, P. Acoustic metamaterial panels for sound attenuation in the 50-1000 Hz regime. Appl. Phys. Lett. 2010, 96, 041906. [CrossRef]

12. Yang, M.; Ma, G.; Yang, Z.; Sheng, P. Coupled membranes with doubly negative mass density and bulk modulus. Phys. Rev. Lett. 2013, 110, 134301. [CrossRef] [PubMed]

13. Sui, N.; Yan, X.; Huang, T.Y.; Xu, J.; Yuan, F.G.; Jing, Y. A lightweight yet sound-proof honeycomb acoustic metamaterial. Appl. Phys. Lett. 2015, 106, 171905. [CrossRef]

14. Li, S.; Mao, D.; Huang, S.; Wang, X. Enhanced transmission loss in acoustic materials with micro-membranes. Appl. Acoust. 2018, 130, 92-98. [CrossRef]

15. Huang, T.Y.; Shen, C.; Jing, Y. Membrane- and plate-type acoustic metamaterials. J. Acoust. Soc. Am. 2016, 139, 3240-3250. [CrossRef] [PubMed]

16. Ma, F.; Huang, M.; Wu, J. Ultrathin lightweight plate-type acoustic metamaterials with positive lumped coupling resonant. J. Appl. Phys. 2017, 121, 015102. [CrossRef]

17. Ma, F.; Huang, M.; Wu, J. Acoustic metamaterials with synergetic coupling. J. Appl. Phys. 2017, 122, 215102. [CrossRef]

18. Ma, F.; Huang, M.; Xu, Y.; Wu, J. Bilayer synergetic coupling double negative acoustic metasurface and cloak. Sci. Rep. 2018, 8, 5906. [CrossRef]

19. Ma, F.; Huang, M.; Xu, Y.; Wu, J. Bilayer plate-type acoustic metamaterials with Willis coupling. J. Appl. Phys. 2018, 123, 035104. [CrossRef]

20. Varanasi, S.; Bolton, J.S.; Siegmund, T.H. Experiments on the low-frequency barrier characteristics of cellular metamaterial panels in a diffuse sound field. J. Acoust. Soc. Am. 2017, 141, 602-610. [CrossRef] [PubMed]

21. Ang, L.Y.L.; Koh, Y.K.; Lee, H.P. Plate-type acoustic metamaterial with cavities coupled via an orifice for enhanced sound transmission loss. Appl. Phys. Lett. 2018, 112, 051903. [CrossRef]

22. Ang, L.Y.L.; Koh, Y.K.; Lee, H.P. A note on the viscous boundary layer in plate-type acoustic metamaterials with an internal tonraum resonator. Appl. Acoust. 2018, 140, 160-166. [CrossRef]

23. Langfeldt, F.; Wolfgang, G.; von Estorff, O. Enhancing the low-frequency noise reduction of a double wall with membrane-type acoustic metamaterials. In Proceedings of the 45th International Congress and Exposition on Noise Control Engineering (INTER-NOISE 2016), Hamburg, Germany, 21-24 August 2016; Kropp, W., Ed.; Deutsche Gesellschaft Fuer Akustik: Hamburg, Germany, 2016; pp. 3413-3424.

24. Ma, G.; Fan, X.; Sheng, P.; Fink, M. Shaping reverberating sound fields with an actively tunable metasurface. Proc. Natl. Acad. Sci. USA 2018, 115, 6638-6643. [CrossRef] [PubMed]

25. Varanasi, S.; Bolton, J.S.; Siegmund, T.H.; Cipra, R.J. The low-frequency performance of metamaterial barriers based on cellular structures. Appl. Acoust. 2013, 74, 485-495. [CrossRef]

26. Ang, L.Y.L.; Koh, Y.K.; Lee, H.P. Plate-type acoustic metamaterials: Evaluation of a large-scale design adopting modularity for customisable acoustical performance. Appl. Acoust. 2019, 149, 156-170. [CrossRef]

27. ASTM. ASTM E90-09: Standard Test Method for Laboratory Measurement of Airborne Sound Transmission Loss of Building Partitions and Elements; ASTM International: West Conshohocken, PA, USA, 2009. [CrossRef]

28. ASTM. ASTM E2235-04: Standard Test Method for Determination of Decay Rates for Use in Sound Insulation Test Methods; ASTM International: West Conshohocken, PA, USA, 2012. [CrossRef]

29. ASTM. ASTM E413-10: Classification for Rating Sound Insulation; ASTM International: West Conshohocken, PA, USA, 2010. [CrossRef] 
30. ASTM. ASTM E1332-16: Standard Classification for Rating Outdoor-Indoor Sound Attenuation; ASTM International: West Conshohocken, PA, USA, 2016. [CrossRef]

31. Fahy, F.J.; Gardonio, P. Transmission of Sound Through Partitions. In Sound and Structural Vibration: Radiation, Transmission, and Response, 2nd ed.; Academic Press: Cambridge, MA, USA, 2007; Chapter 5, pp. 277-373.

(c) 2019 by the authors. Licensee MDPI, Basel, Switzerland. This article is an open access article distributed under the terms and conditions of the Creative Commons Attribution (CC BY) license (http:/ / creativecommons.org/licenses/by/4.0/). 